\title{
ATGL promotes the proliferation of hepatocellular carcinoma cells via the p-AKT signaling pathway
}

\author{
Meiling Liư ${ }^{1}$, Xuegao $\mathrm{Yu}^{2}$, Liying Lin ${ }^{1}$, Jiankai Deng ${ }^{2}$, Kanglong Wang ${ }^{3}$, Baochang $\mathrm{Su}^{5}$, \\ Yong Xia ${ }^{1}$, Xiaohua Tang ${ }^{1}$, Honghai Hong ${ }^{1,4}$.
}

1. Department of Clinical Laboratory, The Third Affiliated Hospital of Guangzhou Medical University, Guangzhou, Guangdong, China.

2. Department of Laboratory Medicine, The First Affiliated Hospital of Sun Yat-Sen University, Guangzhou, Guangdong, China.

3. Department of Blood Transfusion, The Sixth Affiliated Hospital of Sun Yat-Sen University, Guangzhou, Guangdong, China.

4. Department of Pathology and Laboratory Medicine, Indiana University School of Medicine, Indianapolis, Indiana.

5. Department of Blood Transfusion, The First Affiliated Hospital of Jinan University, Guangzhou, China

\begin{abstract}
Abnormal metabolism, including abnormal lipid metabolism, is a hallmark of cancer cells. Some studies have demonstrated that the lipogenic pathway might promote the development of hepatocellular carcinoma (HCC). However, the role of adipose triglyceride lipase (ATGL) in hepatocellular carcinoma cells has not been elucidated. We evaluated the function of ATGL in hepatocellular carcinoma using methyl azazolyl blue and migration assay through overexpression of ATGL in HepG2 cells. Quantitative reverse-transcription polymerase chain reaction and Western blot analyses were used to assess the mechanisms of ATGL in hepatocellular carcinoma. In the current study, we first constructed and transiently transfected ATGL into hepatocellular carcinoma cells. Secondly, we found that ATGL promoted the proliferation of hepatoma cell lines via upregulating the phosphorylation of AKT, but did not affect the metastatic ability of HCC cells. Moreover, the p-AKT inhibitor significantly eliminated the effect of ATGL on the proliferation of hepatoma carcinoma cells. Taken together, our results indicated that ATGL promotes hepatocellular carcinoma cells proliferation through upregulation of the AKT signaling pathway.
\end{abstract}

Keywords: ATGL, hepatocellular carcinoma cells, p-AKT signaling pathway, tumor proliferation.

This is the author's manuscript of the article published in final edited form as:

Liu, M., Yu, X., Lin, L., Deng, J., Wang, K., Su, B., Xia, Y., Tang, X., \& Hong, H. (2019). ATGL promotes the proliferation of hepatocellular carcinoma cells via the $\mathrm{p}$-AKT signaling pathway. Journal of Biochemical and Molecular Toxicology, 33(11), e22391. https://doi.org/10.1002/jbt.22391 


\section{INTRODUCTION}

The occurrence, development, and metastasis of tumors are closely related to fat and lipids. $\underline{\mathbf{1}}, \underline{\mathbf{2}}$ It is reported that serum lipid abnormalities are one of the high risk factors for breast cancer, prostate cancer, and liver cancer, $\underline{\mathbf{3}}, \underline{\mathbf{4}}$ but the exact mechanism is not yet clear. The triacylglycerols (TGs) in the cell exist in human adipose tissue in the form of lipid droplets. When hungry, TGs, stored in fat cells, are hydrolyzed to glycerol and fatty acid, and then perform $\beta$ oxidation to provide energy for the body. The hydrolysis of TGs to glycerol and fatty acid requires at least three lipolytic enzymes. $\underline{\mathbf{5}}, \underline{\mathbf{6}}$ Adipose triglyceride lipase (ATGL) catalyzes the initial step of lipolysis, converting TGs to diacylglycerols (DGs); hormone-sensitive lipase is mainly responsible for the hydrolysis of DGs to monoacylglycerols (MGs) and MG lipase (MGL) hydrolyzes MGs.7 ATGL is the newest member of the lipolytic enzyme trio. The enzyme, first described in 2004, belongs to the family patatin domain-containing proteins including nine human and eight murine members. $\underline{\mathbf{8}}, \underline{9}$ However, the role of ATGL in hepatocellular carcinoma cells is not yet clear. The aim of this study was to study the effect of ATGL on hepatocellular carcinoma cells by silencing or overexpression of ATGL, and to further elucidate its molecular mechanism, providing a theoretical basis and experimental data for the treatment of HCC with ATGL as a target in future.

\section{MATERIALS AND METHODS}

\subsection{CELL CULTURE}

HepG2 cells were from the existing cell lines in our laboratory. Hep3B was purchased from ATCC. Dulbecco's modified Eagle's medium (DMEM) $+10 \%$ fetal bovine serum was cultured in an incubator at $37^{\circ} \mathrm{C}$ and $5 \% \mathrm{CO}_{2}$. The logarithmic phase cells were taken for follow-up experiments.

\subsection{ANTIBODIES AND REAGENTS}

Competent bacteria DH5 a was purchased from Takara for overexpression of ATGL plasmid construction. pcDNA3.1+ was purchased from Invitrogen as a vector for the over expressing ATGL plasmid. Lysogeny broth-related reagents were purchased from Beijing Ding. AKT (catalogue: 4691), p-AKT (catalogue: 9271) and ATGL (catalogue: 2439) 
antibodies were purchased from CST, and $\beta$-actin antibody was purchased from Sigma. The p-AKT inhibitor MK2206 (catalogue: HY-10385) was purchased from MCE. Two methyl sulfoxide (dimethyl sulfoxide, DMSO), four methyl azazolyl blue (MTT) and $4 \%$ polyoxymethylene (paraformaldehyde) were purchased from Beijing Ding Guo company; the ECL chemiluminescence kit was bought from Beijing Pulilai. The transfection reagent Lipo2000 was purchased from Invitrogen.

\subsection{WESTERN BLOT ANALYSIS}

Proteins were separated by sodium dodecyl sulfate polyacrylamide gel electrophoresis and transferred onto a polyvinylidene fluoride membrane. The membranes were incubated in $5 \%$ nonfat dry milk, followed by incubation with a primary antibody against ATGL (1:1000), AKT (1:1000), p-AKT (1:1000), $\beta$-actin (1:10000) and a secondary antibody. Membranes were developed using Clarity Western ECL.

\subsection{CONSTRUCTION OF ATGL-PCDNA3.1 + OVEREXPRESSED PLASMIDS}

1) Primer design

The cDNA sequence of human ATGL was obtained from PubMed, and then ATGL primers were designed according to pcDNA3.1 (+) carrier information. The list of primer sequences is shown in Table 1 .

Table 1. Primer sequences of ATGL PCR produces

$\begin{array}{lll}\text { Primer name } & \text { Primer sequences } & \begin{array}{l}\text { Restric } \\ \text { site }\end{array} \\ \begin{array}{ll}\text { Forward } \\ \text { primer }\end{array} & 5^{\prime} \text {-CCCAAGCTT ATGTTTCCCCCGCGAGAAGACG -3' } & \text { HindIII } \\ \begin{array}{l}\text { Reverse } \\ \text { primer }\end{array} & \begin{array}{l}5^{\prime} \text {-CCGGAATTC CAGCCCCAGGGCCCCGATCACGG- } \\ \text { 3' }\end{array} & \text { EcoRI }\end{array}$


Abbreviations: ATGL, adipose triglyceride lipase; qRT-PCR, quantitative reversetranscription polymerase chain reaction.

2) PCR fishing of target gene and enzyme cut and connection

cDNA was used as a template from the hepatoma cell HepG2. The ATGL gene was extracted using PCR by specific primers, and then used for enzyme digestion, linkage, and identification.

3) Transfection of ATGL-overexpression plasmid

The transfection reagent was Lipo2000 (Invitrogen company). The detailed operation steps are described in the instruction manual. In brief, after transfection for 4 to 6 hours, the cells were converted to the normal culture medium, and the protein or RNA was analyzed at different time points.

\subsection{DETERMINATION OF CELL PROLIFERATION ACTIVITY BY MTT ASSAY}

The HepG2 or Hep3B cells were inoculated in 96 plates transiently transfected with ATGL plasmid or siRNA for ATGL. MTT working fluid was added (the concentration of $5 \mathrm{mg} / \mathrm{mL}$ ) and cultured for 4 hours followed by removing the supernatant. $250 \mu \mathrm{L}$ DMSO was added to each hole and determined with an instrument in $570 \mathrm{~nm}$. All the experiments were repeated three times.

\subsection{QUANTITATIVE REVERSE-TRANSCRIPTION POLYMERASE CHAIN REACTION}

Quantitative reverse-transcription polymerase chain reaction (RT-PCR) was used by means of an $\mathrm{ABI}$ fluorescence quantitative PCR instrument. The reagent was SYBR Green I from Takara. The primers were designed by a Shanghai-based company, as shown in Table 2 . 
Table 2. $\mathrm{qRT}$-PCR sequences

$\begin{array}{lll}\text { Gene } & \text { Forward primers } & \text { Reverse primers } \\ \text { ATGL } & \text { 5'-GGCCAGACCCAGCTTCTTC-3' }^{\prime} & \text { 5'-GCCAGGCCTCTGTGAGC-3' } \\ \text { B-Actin } & 5^{\prime} \text {-GCACTCTTCCAGCCTTCCTT-3' } & \text { 5'-GTTGGCGTACAGGTCTTTGC-3' }\end{array}$

Abbreviations: ATGL, adipose triglyceride lipase; qRT-PCR, quantitative reversetranscription polymerase chain reaction.

The Real-time PCR reaction conditions are as follows:

Two step method: $95^{\circ} \mathrm{C}, 30 \mathrm{~s} ; 1$ cycle. PCR reaction, $95^{\circ} \mathrm{C}, 5 \mathrm{~s} ; 60^{\circ} \mathrm{C}, 15 \mathrm{~s} ; 45$ cycles; dissolution, $95^{\circ} \mathrm{C}, 5 \mathrm{~s} ; 65^{\circ} \mathrm{C}, 20 \mathrm{~s} ; 95^{\circ} \mathrm{C}, 5 \mathrm{~s} ; 1$ cycle. After the end of PCR, the relative standard analysis method was used to correct the standard curve, and finally the transcriptional level of each gene was calculated.

\subsection{MIGRATION ASSAY}

Migration of HepG2 cells were examined using 24-well Boyden chambers (Corning, Corning, NY) with $8 \mu \mathrm{m}$ inserts. Briefly, the serum-starved cells were trypsinized and plated at the upper chamber at $2 \times 10^{4}$ cells per well, together with serum-free DMEM and $10 \%$ fetal bovine serum placed in the lower chamber. The cells were then allowed to migrate for 12 hours at $37^{\circ} \mathrm{C}$. After the incubation, nonmigrating cells were removed from the upper chamber with a cotton swab, and the membrane inserts were stained with crystal violet solution.

\subsection{STATISTICAL ANALYSIS}

T-test was used for comparative analysis, and $P$ less than .05 was significantly different. All the experiments were repeated three times. 


\section{RESULTS}

\subsection{ATGL CONSTRUCTION AND OVEREXPRESSION IN HEPATOCELLULAR CARCINOMA CELLS}

To study the effect of ATGL on hepatocellular carcinoma, we first constructed the ATGL plasmid. HepG2 cell cDNA was used as template for amplication of ATGL CDS sequence by PCR. The result for the ATGL fragment is shown in Figure $1 \mathrm{~A}$. To verify the successful overexpression of ATGL plasmid in hepatoma cells, HepG2 was transfected with the ATGL plasmid, and validated by Western blot analysis and real-time fluorescent quantitative PCR. The results showed that the ATGL plasmid was successfully transfected into hepatocellular carcinoma ( $\mathrm{HCC}$ ) cells, and highly expressed (Figure 1 $\mathrm{B}$ and $1 \mathrm{C})$.

Figure 1.

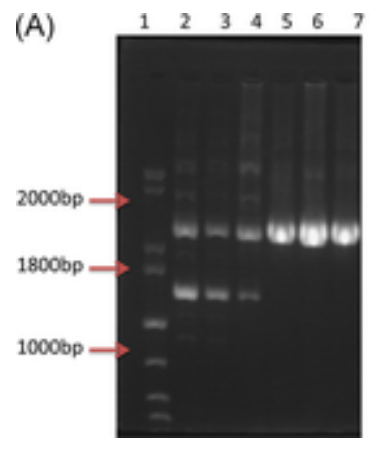

(C)

(B)
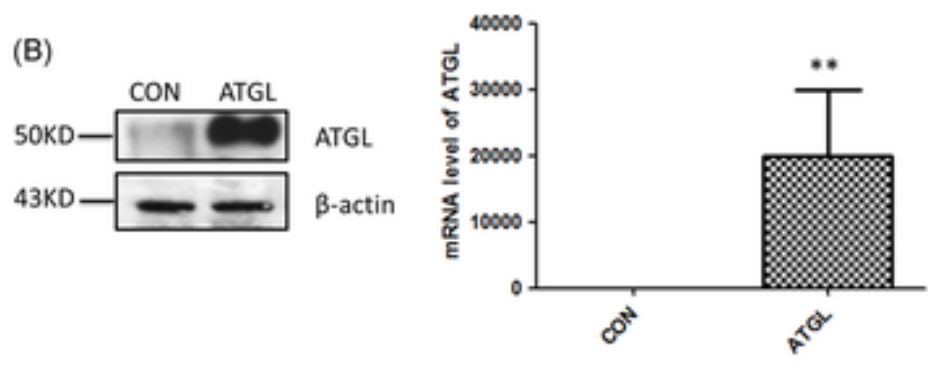

ATGL construction and overexpression in hepatocellular carcinoma cells. A, Agarose electrophoresis was used to identify the PCR amplification products of ATGL. 1, DNA maker; $2-7$, the annealing temperature is $55,58,61,64,67,70^{\circ} \mathrm{C}$ in turn. $\mathrm{B}, \mathrm{ATGL}$ was transfected into HepG2 cells after 24 hours and protein was collected. Western blot analysis was used to detect the ATGL level with $\beta$-Actin as the loading control. $C$, The transcriptional level of ATGL was detected by qRT-PCR. ATGL was transfected into HepG2 cells for 24 hours and RNA was extracted. ${ }^{* *} P<.001$. ATGL, adipose triglyceride 
lipase; CON, control; PCR, polymerase chain reaction; qRT-PCR, quantitative reversetranscription PCR

\subsection{ATGL PROMOTES THE PROLIFERATION OF HEPATOCELLULAR CARCINOMA CELLS}

To study the effect of ATGL on hepatocellular carcinoma, we overexpressed ATGL in the hepatoma cell line HepG2 and detected the proliferation ability of HepG2 by MTT assay. MTT experiments showed that ATGL significantly promoted the proliferation of hepatocellular carcinoma (Figure $\underline{2}$ A). To fully illustrate the role of ATGL in promoting the proliferation of hepatoma cells, we also tested the effect of ATGL on the Hep3B cell line. Consistent with the results on HepG2, overexpression of ATGL also promoted the proliferation of Hep3B (Figure 2B). Furthermore, overexpression of ATGL increased the proliferation of HepG2 by colony formation assay (Figure S1). Moreover, knocking down with siRNA for ATGL inhibited cell proliferation (Figure S2), which fully demonstrated that ATGL promoted the proliferation of HCC.

\section{Figure 2.}
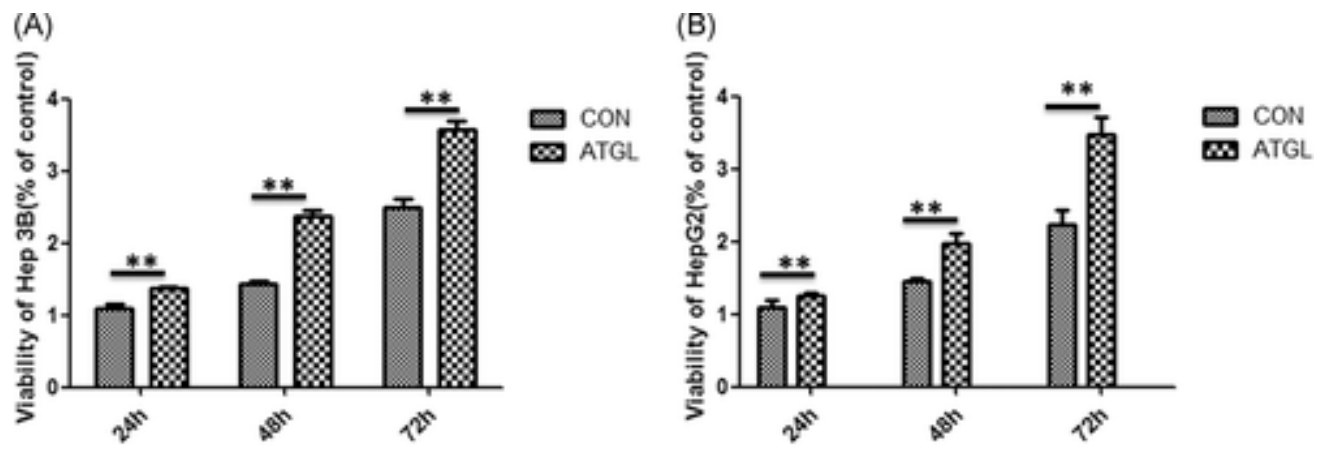

ATGL promotes the proliferation of hepatocellular carcinoma cells. A, MTT assay was used to detect the proliferation of Hep3B cells. Hep3B cells were transfected with ATGL plasmid for 24,48 and 72 hours, and then we detected the proliferative ability of Hep3B cells. ${ }^{* *} P<.01$. B, MTT assay was used to detect the proliferation of HepG2 cells. HepG2 cells were transfected with ATGL plasmid for 24,48 , and 72 hours, and then we detected the proliferative ability of HepG2 cells. ATGL, adipose triglyceride lipase; MTT, methyl azazolyl blue. ${ }^{* *} P<.01$ 


\subsection{ATGL DOES NOT AFFECT THE MIGRATION OF HEPATOMA CELLS}

To evaluate the effect of ATGL on the migration ability of hepatoma cells, we also carried out the Migration assay. The migration assay involves experimentation with the Boyden Transwell cell. After the treated cells were digested, the cells were planted in the Boyden Transwell compartment with serum-free medium and $10 \%$ fetal bovine serum under the chamber. The cells in the chamber migrated through the chamber hole to the lower chamber, and the greater the cell number that passed through the chamber, the stronger the migration energy. After transfection of ATGL plasmid for 24 hours, the cells were digested and cultured in the Boyden Transwell, and the number of cells passing through the Boyden Transwell was detected at different times. Migration assay results showed that overexpression of ATGL did not affect the migration ability of HepG2 cells (Figure $\underline{3} A$ and $\underline{3} B$ ), compared with the control group.

\section{Figure 3.}

(A)

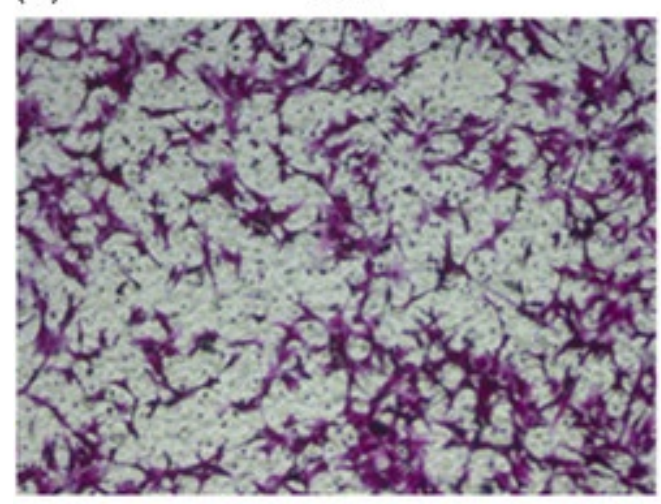

(B)

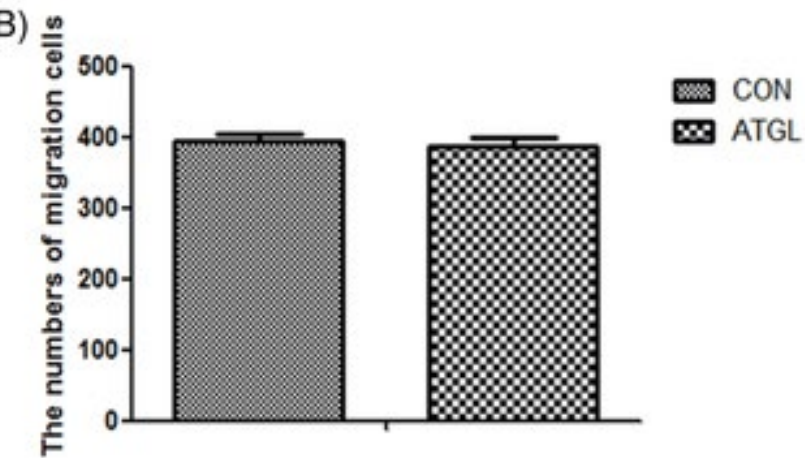

ATGL

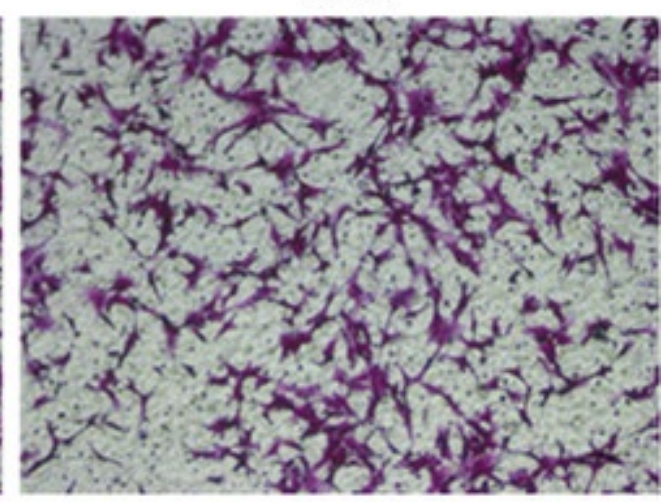

CON

ATGL

The metastasis of the hepatocellular carcinoma cell. A, Migration assay was used to detect the effect of ATGL on the migration of HepG2 cells. Transfection of ATGL plasmid 
for 24 hours, the cells were digested and cultured in Boyden Transwell chamber. B, The number of HepG2 cells passing through the Boyden Transwell chamber was counted. ATGL, adipose triglyceride lipase; CON, control

\subsection{ATGL UPREGULATED THE LEVEL OF PHOSPHORYLATION OF AKT}

The p-AKT signaling pathway plays an important role in tumorigenesis, proliferation, metastasis, and invasion. To further study the molecular mechanism of ATGL promoting the proliferation of HCC, we detected the p-AKT signaling pathway. We found that the overexpression of ATGL significantly upregulated the level of phosphorylation of AKT (Figure 4), indicating that ATGL promotes the proliferation of hepatoma carcinoma cells by regulating the p-AKT signaling pathway. To further verify that ATGL promotes the proliferation of hepatoma carcinoma cells through the AKT signaling pathway, we used the p-AKT inhibitor to treat cells and then performed the MTT assay. The results showed that the p-AKT inhibitor significantly eliminated the effect of ATGL on the p-akt level and proliferation of hepatoma carcinoma cells (Figures $\underline{\mathbf{4}}$ and $\underline{\mathbf{5}}$ ).

Figure 4.

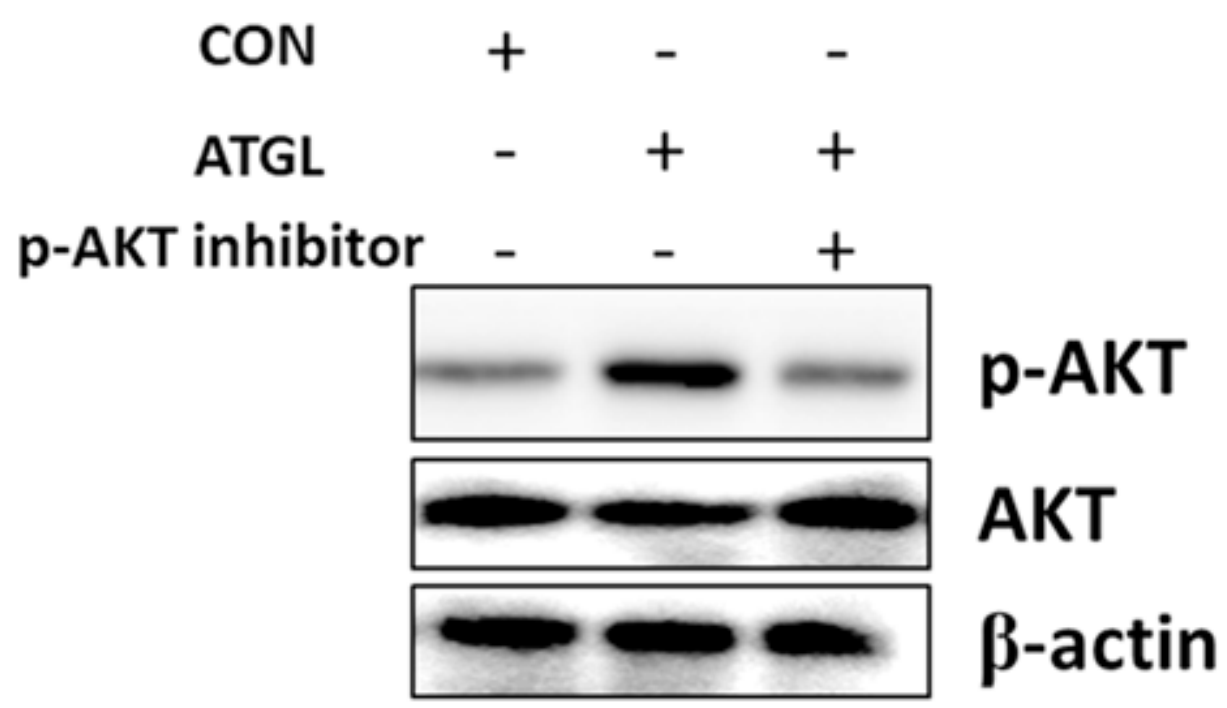

ATGL upregulated the level of phosphorylation of AKT. ATGL promotes the phosphorylation of AKT. ATGL plasmid was overexpressed in HepG2 cells for 24 hours, and the $p$-AKT and AKT levels were detected by Western blot analysis. $\beta$-Actin as loading control. ATGL, adipose triglyceride lipase; CON, control. 
Figure 5.
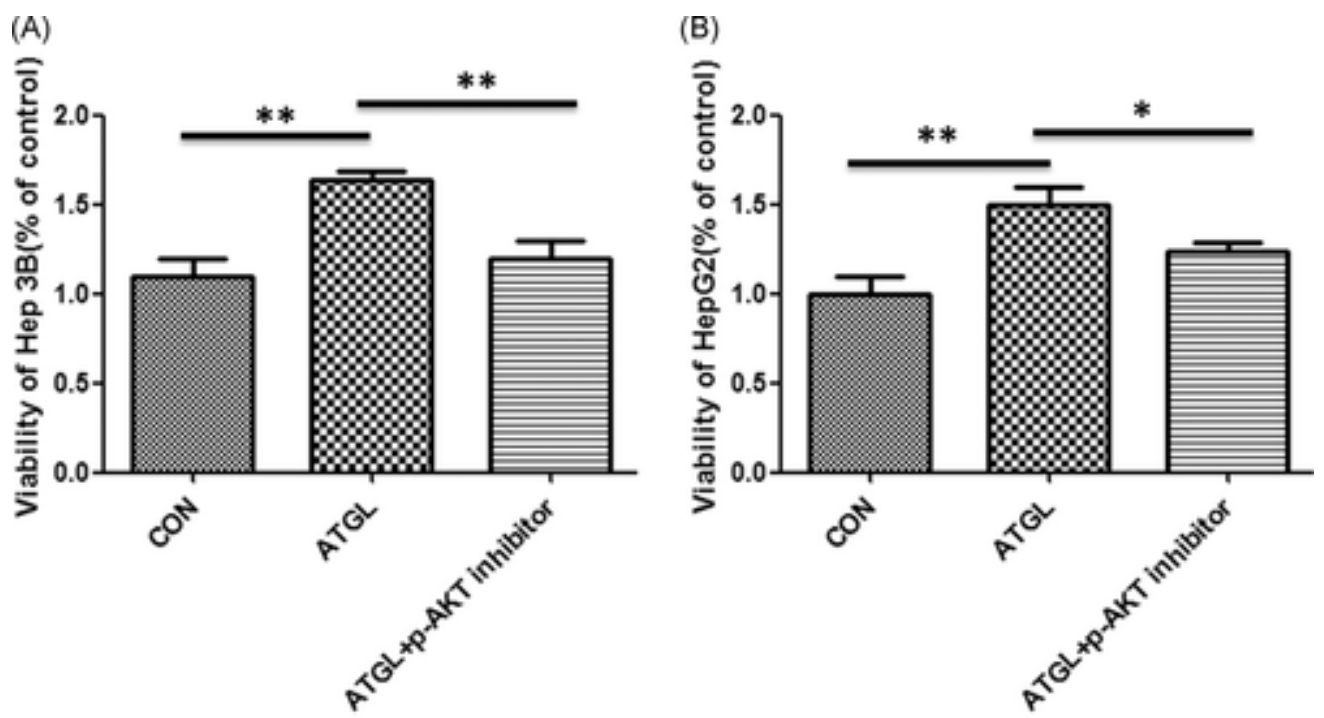

ATGL promotes the proliferation of hepatocellular carcinoma cells via the $p$-AKT signaling pathway. A, MTT assay was used to detect the proliferation of Hep3B cells. Hep3B cells were transfected with ATGL plasmid for 24 hours, and then the p-AKT inhibitor was treated for 12 hours. B, MTT assay was used to detect the proliferation of HepG2 cells. HepG2 cells were transfected with ATGL plasmid for 24 hours, and then the p-AKT inhibitor was treated for 12 hours. ${ }^{*} P<.05 ;{ }^{* *} P<.001$. ATGL, adipose triglyceride lipase; CON, control; MTT, methyl azazolyl blue.

\section{DISCUSSION}

The intracellular fat is mainly in the form of lipid droplets. ATGL is the first enzyme for fat degradation, which degrades fat to monoacylglycerols (MGs) and starts the fat mobilization.10, 11 It is found that abnormal metabolism of lipids is closely related to the biological processes such as tumor occurrence, development, metastasis, and so on.3, 4 However, the role of ATGL in regulating lipid metabolism and the tumor is still not clear. To study the role of ATGL in hepatocellular carcinoma cells, we first constructed the overexpression plasmid of ATGL. The pcDNA3.1+ vector is the most commonly used expression vector, highly expressed in eukaryotic cells, and is suitable for various gene 
expressions. So we chose pcDNA3.1+ as a vector to construct the ATGL overexpression plasmid.

We first extracted RNA from the hepatocellular carcinoma cell HepG2, and reversed to cDNA, as a template to catch the target gene of ATGL. We designed specific primers based on the CDS region of the human ATGL gene on PubMed and added HindIII and EcoRI restriction sites at the ends of the primers. For catching ATGL gene fragments, we designed different annealing temperatures. The agarose electrophoresis results showed that the ATGL target gene fragment could be found from the annealing temperature of $55^{\circ} \mathrm{C}$ to $70^{\circ} \mathrm{C}$. The brightest ATGL band (Figure 1) at the annealing temperature was $67^{\circ} \mathrm{C}$ (Figure 1), indicating that the $67^{\circ} \mathrm{C}$ was the best annealing temperature. Subsequently, we inserted the ATGL gene into the pcDNA3.1+ vector by molecular cloning steps such as enzyme digestion, linkage, and transformation. Furthermore, we transfected the ATGL plasmid into the hepatocellular carcinoma cell line HepG2 and verified its expression in HepG2. We selected Lipo2000 as a transfection agent and transfected the ATGL plasmid for 24 hours to collect RNA and protein. Quantitative RT$\mathrm{PCR}$ and Western blot analyses were used to evaluate the transcriptional and protein level of ATGL plasmid in HepG2. After 24 hours transfection of ATGL, the transcriptional level of ATGL was nearly 20000 times as high as that of the control group, indicating that the ATGL plasmid was highly expressed in the hepatoma cell HepG2, which established the basis for the next step of research on ATGL. In addition, the ATGL protein level was highly expressed, which was consistent with the transcriptional level.

To verify the effect of ATGL on tumor, we overexpressed ATGL in the hepatoma cell line HepG2, and MTT method was used to detect the growth ability of HepG2 cells. We found that the transfection of ATGL plasmids significantly promoted the growth of HepG2 cells (Figure 2). At the same time, we also assessed the function of ATGL in the Hep3B hepatoma cells. The results showed that ATGL also promoted the growth of Hep3B cells, which was consistent with the results of HepG2 cells, indicating that ATGL promoted the growth of tumor cells. Our result is consistent with the results of Zagani R's research,12 which showed that blocking ATGL with the ATGL inhibitor GOS2 or the ATGL small interfering fragment inhibited the growth of non-small-cell lung cancer. But recently, Al- 
Zoughbi et al13 have found that ATGL is lowly expressed in breast cancer, gastric cancer and non-small cell lung cancer, and the absence of ATGL could lead to the occurrence of tumor, indicating that ATGL could induce the tumor to occur and inhibit the proliferation of tumor. the This contradiction need to be studied further. In addition, we also studied the effect of ATGL on the metastasis of HCC. The migration assay showed that ATGL had no effect on the migration of HCC, suggesting that the effect of ATGL on HCC mainly promoted the proliferation of the cancer cell without affecting the migration of the cancer cell. Based on the above phenomenon, we will next focus on the molecular mechanism of ATGL promoting the proliferation of hepatocellular carcinoma.

Tumor growth and proliferation are closely related to the p-AKT signaling pathway.14, 15 Next, we will study whether ATGL promotes tumor growth through the p-AKT signaling pathway. Our results shown that ATGL significantly upregulated the level of phosphorylation of AKT, indicating that ATGL promotes the growth of tumor cells through the p-AKT signaling pathway, which is consistent with the research reported that $p-A K T$ promotes the proliferation of HCC.16, 17

\section{CONCLUSION}

The current paper reveals the role of ATGL in HCC and elucidates its molecular mechanism, which provides a theoretical basis and experimental data for future targeting of ATGL in HCC therapies.

\section{ACKNOWLEDGMENT}

This study was supported by the Laboratory Opening Project of Guangzhou Medical University Students (Grant Numbers: B185004039), the Western Medicine Guide Project of Guangzhou Municipal Health Bureau (Grant numbers: 20171A011312); Guangzhou Traditional Chinese Medicine, integrated traditional Chinese and Western Medicine Science and Technology Project (Grant numbers: 20182A011020) and research project on elite talent training of the Third Affiliated Hospital of Guangzhou Medical University.

\section{CONFLICT OF INTERESTS}

The authors declare that there are no conflicts of interests. 


\section{AUTHOR CONTRIBUTIONS}

M.L., X.Y., and K.W. were responsible for the cell experiment; Y.X. and H.H. were responsible for experimental design and guidance.

\section{REFERENCES}

1. Grundy, S. M. (2004). Obesity, Metabolic Syndrome, and Cardiovascular Disease. The Journal of Clinical Endocrinology \& Metabolism, 89(6), 2595-2600. https://doi.org/10.1210/jc.2004-0372

2. Glass, C. K., \& Witztum, J. L. (2001). Atherosclerosis: The Road Ahead. Cell, 104(4), 503-516. https://doi.org/10.1016/S0092-8674(01)00238-0

3. Hsu, P. P., \& Sabatini, D. M. (2008). Cancer Cell Metabolism: Warburg and Beyond. Cell, 134(5), 703-707. https://doi.org/10.1016/i.cell.2008.08.021

4. Rysman, E., Brusselmans, K., Scheys, K., Timmermans, L., Derua, R., Munck, S., Veldhoven, P. P. V., Waltregny, D., Daniëls, V. W., Machiels, J., Vanderhoydonc, F., Smans, K., Waelkens, E., Verhoeven, G., \& Swinnen, J. V. (2010). De novo Lipogenesis Protects Cancer Cells from Free Radicals and Chemotherapeutics by Promoting Membrane Lipid Saturation. Cancer Research, 70(20), 8117-8126. https://doi.org/10.1158/0008-5472.CAN-09-3871

5. Liu, P., Ying, Y., Zhao, Y., Mundy, D. I., Zhu, M., \& Anderson, R. G. W. (2004). Chinese Hamster Ovary K2 Cell Lipid Droplets Appear to Be Metabolic Organelles Involved in Membrane Traffic. Journal of Biological Chemistry, 279(5), 3787-3792. https://doi.org/10.1074/jbc.M311945200

6. Schaffer, J. E. (2003). Lipotoxicity: When tissues overeat. Current Opinion in Lipidology, 14(3), 281-287.

7. Haemmerle, G., Lass, A., Zimmermann, R., Gorkiewicz, G., Meyer, C., Rozman, J., Heldmaier, G., Maier, R., Theussl, C., Eder, S., Kratky, D., Wagner, E. F., Klingenspor, M., Hoefler, G., \& Zechner, R. (2006). Defective Lipolysis and Altered Energy Metabolism in Mice Lacking Adipose Triglyceride Lipase. Science, 312(5774), 734-737. https://doi.org/10.1126/science.1123965

8. Jocken, J. W. E., Smit, E., Goossens, G. H., Essers, Y. P. G., van Baak, M. A., Mensink, M., Saris, W. H. M., \& Blaak, E. E. (2008). Adipose triglyceride lipase (ATGL) expression 
in human skeletal muscle is type I (oxidative) fiber specific. Histochemistry and Cell Biology, 129(4), 535-538. https://doi.org/10.1007/s00418-008-0386-y

9. Reid, B. N., Ables, G. P., Otlivanchik, O. A., Schoiswohl, G., Zechner, R., Blaner, W. S., Goldberg, I. J., Schwabe, R. F., Chua, S. C., \& Huang, L.-S. (2008). Hepatic Overexpression of Hormone-sensitive Lipase and Adipose Triglyceride Lipase Promotes Fatty Acid Oxidation, Stimulates Direct Release of Free Fatty Acids, and Ameliorates Steatosis. Journal of Biological Chemistry, 283(19), 13087-13099. https://doi.org/10.1074/jbc.M800533200

10. Sunny, N. E., Parks, E. J., Browning, J. D., \& Burgess, S. C. (2011). Excessive Hepatic Mitochondrial TCA Cycle and Gluconeogenesis in Humans with Nonalcoholic Fatty Liver Disease. Cell Metabolism, 14(6), 804-810. https://doi.org/10.1016/j.cmet.2011.11.004

11. Satapati, S., Sunny, N. E., Kucejova, B., Fu, X., He, T. T., Méndez-Lucas, A., Shelton, J. M., Perales, J. C., Browning, J. D., \& Burgess, S. C. (2012). Elevated TCA cycle function in the pathology of diet-induced hepatic insulin resistance and fatty liver. Journal of Lipid Research, 53(6), 1080-1092. https://doi.org/10.1194/jlr.M023382

12.Zagani, R., El-Assaad, W., Gamache, I., \& Teodoro, J. G. (2015). Inhibition of adipose triglyceride lipase (ATGL) by the putative tumor suppressor G0S2 or a small molecule inhibitor attenuates the growth of cancer cells. Oncotarget, 6(29), 28282-28295.

13.Al-Zoughbi, W., Pichler, M., Gorkiewicz, G., Guertl-Lackner, B., Haybaeck, J., Jahn, S. W., Lackner, C., Liegl-Atzwanger, B., Popper, H., Schauer, S., Nusshold, E., Kindt, A. S. D., Trajanoski, Z., Speicher, M. R., Haemmerle, G., Zimmermann, R., Zechner, R., Vesely, P. W., \& Hoefler, G. (2016). Loss of adipose triglyceride lipase is associated with human cancer and induces mouse pulmonary neoplasia. Oncotarget, 7(23), 3383233840. https://doi.org/10.18632/oncotarget.9418

14.Xue, D., Zhou, C., Lu, H., Xu, R., Xu, X., \& He, X. (2016). LncRNA GAS5 inhibits proliferation and progression of prostate cancer by targeting miR-103 through AKT/mTOR signaling pathway. Tumor Biology, 37(12), 16187-16197. https://doi.org/10.1007/s13277-016-5429-8

15. Yang, B., Yan, P., Gong, H., Zuo, L., Shi, Y., Guo, J., Guo, R., Xie, J., \& Li, B. (2016). TWEAK protects cardiomyocyte against apoptosis in a PI3K/AKT pathway dependent manner. American Journal of Translational Research, 8(9), 3848-3860. 
16. Tu, K., Liu, Z., Yao, B., Han, S., \& Yang, W. (2016). MicroRNA-519a promotes tumor growth by targeting PTEN/PI3K/AKT signaling in hepatocellular carcinoma. International Journal of Oncology, 48(3), 965-974. https://doi.org/10.3892/ijo.2015.3309

17.Hsieh, Y.-H., Hsieh, S.-C., Lee, C.-H., Yang, S.-F., Cheng, C.-W., Tang, M.-J., Lin, C.-L., Lin, C.-L., \& Chou, R.-H. (2015). Targeting EMP3 suppresses proliferation and invasion of hepatocellular carcinoma cells through inactivation of PI3K/Akt pathway. Oncotarget, 6(33), 34859-34874. 